\title{
Bilateral movement training promotes axonal remodeling of the corticospinal tract and recovery of motor function following traumatic brain injury in mice
}

\author{
H Nakagawa ${ }^{1,2}$, M Ueno $^{1,2}$, Itokazu $^{1,2,3}$ and T Yamashita ${ }^{*, 1,2}$
}

Traumatic brain injury (TBI) results in severe motor function impairment, and subsequent recovery is often incomplete. Rehabilitative training is considered to promote restoration of the injured neural network, thus facilitating functional recovery. However, no studies have assessed the effect of such trainings in the context of neural rewiring. Here, we investigated the effects of two types of rehabilitative training on corticospinal tract (CST) plasticity and motor recovery in mice. We injured the unilateral motor cortex with contusion, which induced hemiparesis on the contralesional side. After the injury, mice performed either a single pellet-reaching task (simple repetitive training) or a rotarod task (bilateral movement training). Multiple behavioral tests were then used to assess forelimb motor function recovery: staircase, ladder walk, capellini handling, single pellet, and rotarod tests. The TBI + rotarod group performed most forelimb motor tasks (staircase, ladder walk, and capellini handling tests) better than the TBI-only group did. In contrast, the TBI + reaching group did not perform better except in the single pellet test. After the injury, the contralateral CST, labeled by biotinylated dextran amine, formed sprouting fibers into the denervated side of the cervical spinal cord. The number of these fibers was significantly higher in the TBI + rotarod group, whereas it did not increase in the TBI + reaching group. These results indicate that bilateral movement training effectively promotes axonal rewiring and motor function recovery, whereas the effect of simple repetitive training is limited.

Cell Death and Disease (2013) 4, e534; doi:10.1038/cddis.2013.62; published online 7 March 2013

Subject Category: Neuroscience

Traumatic brain injury (TBI) occurs frequently during accidents and is the leading cause of motor function deficit in young adults. ${ }^{1}$ Among acute brain injury patients who require rehabilitation, 55-75\% experience functional impairment of the hemiplegic arm and fingers 3-6 months after injury, and only $5-20 \%$ achieve near-complete functional recovery. ${ }^{2}$ One of the main causes of these impairments is disruption of corticospinal connections, which are important for skilled arm, hand, and finger movements. ${ }^{3}$ Therefore, rewiring the corticospinal tract (CST) after brain injury is considered critical for functional recovery; however, this is limited because the adult central nervous system (CNS) is limited in terms of axonal plasticity and regeneration. We previously used a mouse model of TBI to demonstrate that intraspinal axonal rewiring of the CST on the intact side is critical for recovery. ${ }^{4}$ The intact side CST forms sprouting fibers that grow into the denervated side of the spinal cord and make functional connections with propriospinal and segmental interneurons. This newly formed corticospinal circuit was shown to be necessary for motor recovery.

Rehabilitative therapies can successfully enhance motor recovery after brain injury. ${ }^{5}$ In rodent models, simple repetitive reaching training is often employed. It enhances motor performance after brain injury and induces plastic changes in the motor cortex. ${ }^{6,7}$ However, clinical studies have shown that repetitive upper limb training does not enhance functional recovery of the affected arm and hand in CNS-injured patients. ${ }^{8,9}$ Therefore, the mechanism of recovery and neural reorganization by simple repetitive training in rodents may not be equivalent to that in patients. Whitall et al. ${ }^{10}$ moved beyond unilateral training and demonstrated that bilateral movements paced by an auditory metronome led to improved paretic upper limb recovery in patients. Thus, bilateral movement training might be a promising method for recovering upper limb function, but the basic neuronal mechanism of this phenomenon is unclear. ${ }^{11-13}$ Hence, understanding the neural basis of recovery following bilateral training is important.

In this study, we compared the effects of unilateral repetitive and bilateral movement on motor function recovery in a mouse model of TBI and explored the mechanism of recovery, with a focus on CST rewiring. Our data indicate that bilateral movement training effectively promotes axonal rewiring and motor function recovery, whereas simple repetitive training achieved only limited improvement.

\footnotetext{
${ }^{1}$ Department of Molecular Neuroscience, Graduate School of Medicine, Osaka University, Suita-shi, Osaka, Japan; ${ }^{2}$ Core Research for Evolutional Science and Technology (CREST), Japan Science and Technology Agency (JST), Chiyoda-ku, Tokyo, Japan and ${ }^{3}$ Department of Neurology, Graduate School of Medicine, Kyoto University, Sakyo-ku, Kyoto, Japan

*Corresponding author: T Yamashita, Department of Molecular Neuroscience, Graduate School of Medicine, Osaka University, 2-2 Yamadaoka, Suita-shi, Osaka 565-0871, Japan. Tel: +81 66879 3661; Fax: +81 66879 3669; E-mail: yamashita@molneu.med.osaka-u.ac.jp

Keywords: traumatic brain injury; rehabilitative training; functional recovery; corticospinal tract

Abbreviations: TBI, traumatic brain injury; CST, corticospinal tract; CNS, central nervous system; PFA, paraformaldehyde; BDA, biotinylated dextran amine; PRV, pseudorabies virus; CPGs, central pattern generators; EGFP, enhanced green fluorescent protein; BDNF, brain-derived neurotrophic factor; NeuN, neuronal nuclei; CMV, cytomegalovirus

Received 27.9.12; revised 10.1.13; accepted 06.2.13; Edited by A Verkhratsky
} 


\section{Results}

Histological assessment of cortical lesions. We employed a traumatic injury model in mice and investigated the effects of two different rehabilitative methods, reaching and rotarod training, on motor recovery after the injury. In our model, the unilateral forelimb area of the motor cortex was fully destroyed in which the CST is mostly degenerated (Figures 1a and 4a). ${ }^{4,14}$ To confirm the absence of corticospinal neurons in this model, pseudorabies virus (PRV) expressing enhanced green fluorescent protein (EGFP), a retrograde transsynaptic viral tracer, ${ }^{15}$ was injected into the forelimb muscle, and corticospinal neurons were examined for the presence of EGFP. In the sham group, most EGFPpositive neurons were found in layer $\mathrm{V}$ of the motor area contralateral to the injection side (Figures $1 \mathrm{~b}$ and $\mathrm{c}$ ). In the $\mathrm{TBI}, \mathrm{TBI}$ + reaching, and TBI + rotarod groups, EGFP-positive neurons were absent in the contralateral motor area, but they were found in layer $\mathrm{V}$ of the ipsilateral motor area (Figures $1 \mathrm{~d}-\mathrm{i}$ ), confirming that ipsilesional CST mostly degenerated in this model. Lesion volume was measured 34 days after TBI and was not significantly different among the TBI $(28.8 \pm 1.1 \%), \mathrm{TBI}+$ reaching $(27.2 \pm 1.5 \%)$, and $\mathrm{TBI}+$ rotarod groups $(29.9 \pm 0.8 \%)$ (Figure $1 \mathrm{j})$.

Bilateral movement enhances motor test performance after TBI. We used two different rehabilitative methods, reaching and rotarod training after $\mathrm{TBI}$, and investigated the effects on motor function recovery (Figure 2). Impaired forelimb motor performance was assessed with the staircase
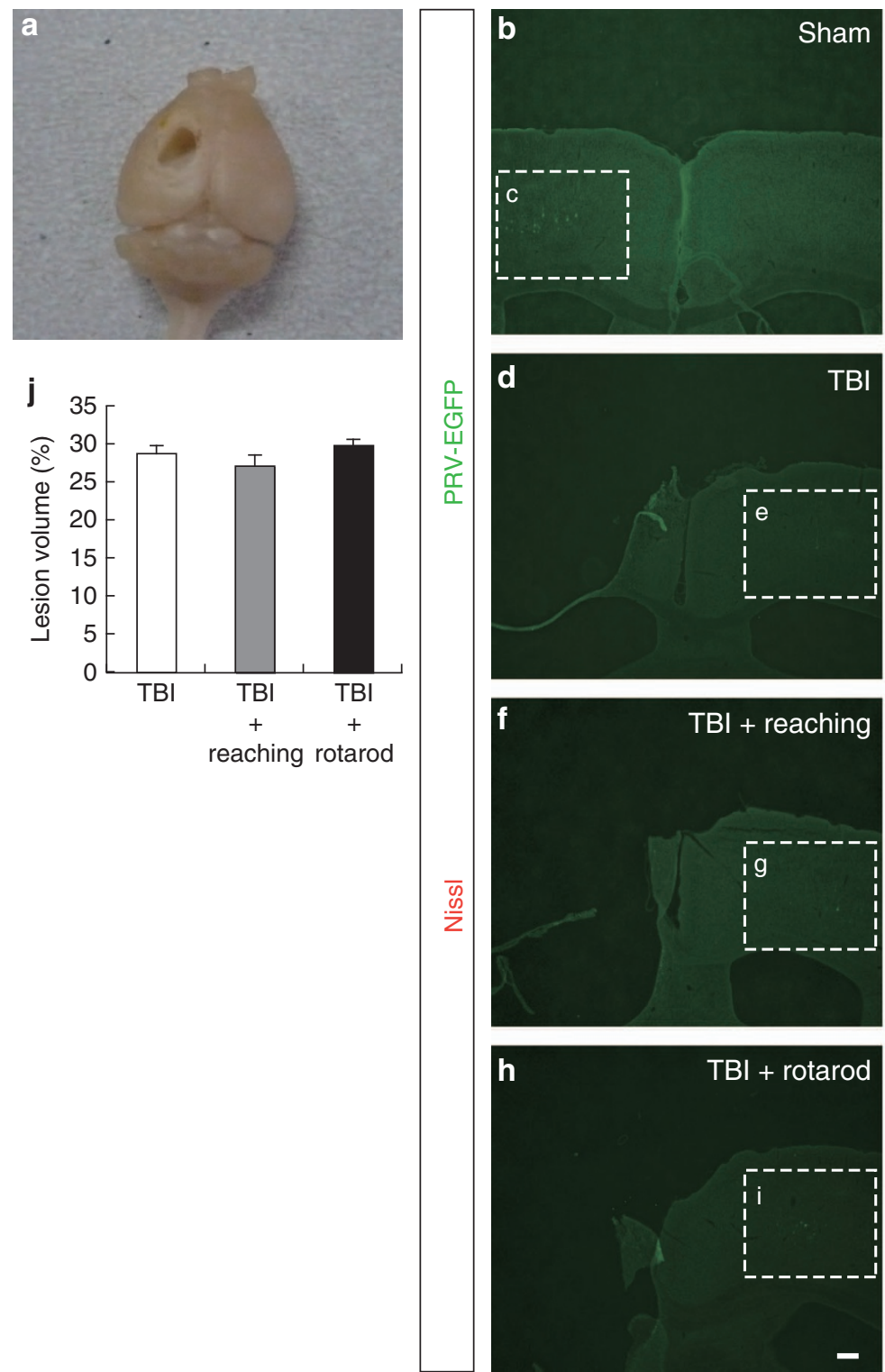
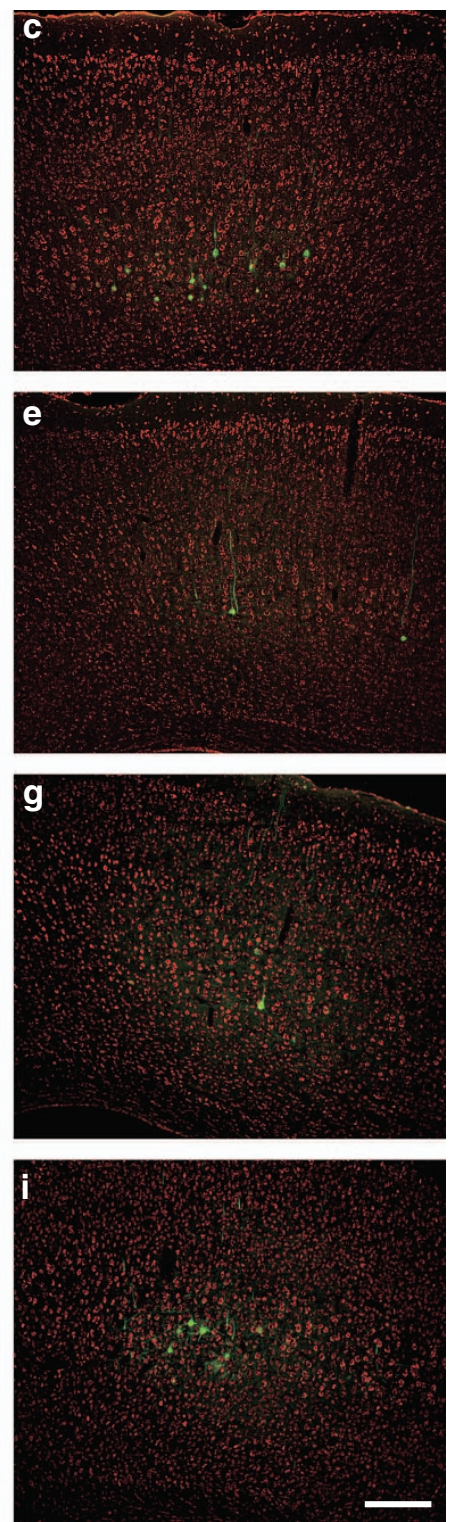

Figure 1 TBI mouse model. (a) Unilateral sensorimotor cortex contusion by TBI (34 days after injury). (b-i) Immunohistochemistry for anti-GFP and Nissl, which were injected with PRV-EGFP to the impaired forelimb, in the cortex of the sham ( $\mathbf{b}$ and $\mathbf{c}), \mathrm{TBI}(\mathbf{d}$ and $\mathbf{e}), \mathrm{TBI}+$ reaching (f and $\mathbf{g})$, and TBI + rotarod groups (h and i) at day 39 . (c, e, $\mathbf{g}$ and i) Magnified views of EGFP-positive layer V neurons in the contralateral (sham) and ipsilateral (TBI, TBI + reaching, and TBI + rotarod groups) cortex to the impaired side, respectively. Scale bars: $200 \mu \mathrm{m}$. (j) Percentage of lesion volume in the injured hemisphere 


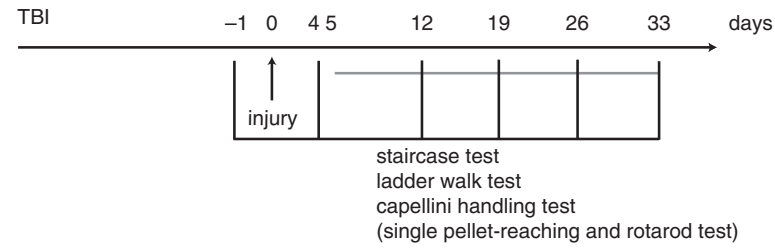

b

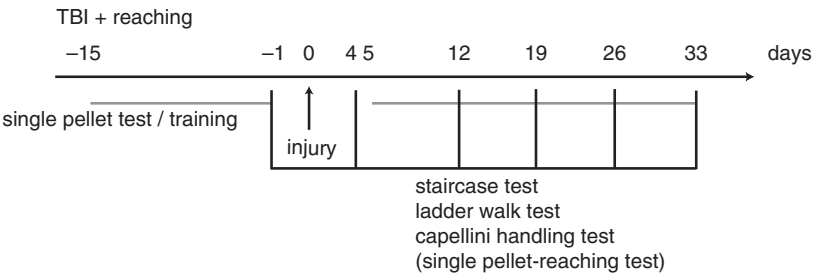

C

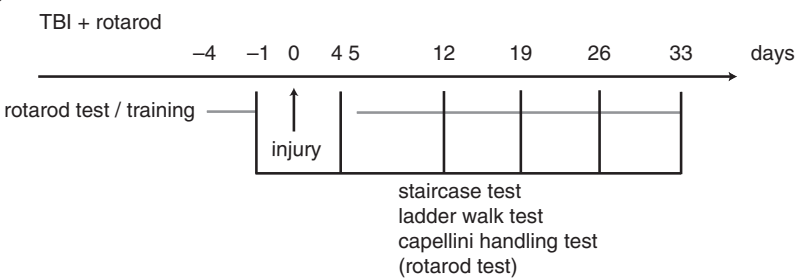

Figure 2 Experiment time course. (a-c) Behavioral tests (staircase test, ladder walk test, and capellini handling test) were performed on days 4 (before training), $12,19,26$, and 33 . Single pellet and rotarod tests were performed on days 4 and 33 . Rehabilitative training was started 5 days after brain injury. (a) TBI; (b) TBI + reaching; and (c) TBI + rotarod

test, ladder walk test, and capellini handling test. These tests were administered 4 days after TBI and then once a week for 4 weeks. The staircase test was used to assess skilled forelimb reaching, which is severely impaired after TBI. ${ }^{16,17}$ In this test, the $\mathrm{TBI}$ + rotarod group performed the forelimb reaching task better than the TBI-only group did (Figure 3a; two-way analysis of variance (ANOVA), $P<0.05$ ). However, we did not observe a significant difference between the $\mathrm{TBI}+$ reaching and $\mathrm{TBI}$-only groups (Figure $3 \mathrm{a}$ ).

The ladder walk test was conducted to assess skilled walking by quantifying the foot fault score,${ }^{18}$ which evaluates digit, wrist, and forelimb motion. We observed an increased number of missed steps with the affected forelimb at day 4 after the injury, which gradually decreased in all the TBI groups (Figure 3b). Statistical analysis revealed a significantly greater recovery in the $\mathrm{TBI}$ + rotarod group compared with the $\mathrm{TBI}$ + reaching and TBI-only groups 33 days after the injury (Figure $3 b$; two-way ANOVA followed by Tukey-Kramer test, $P<0.05$ ).

The capellini handling test is a simple and quantitative way to estimate paw and digit function after motor cortex damage. ${ }^{19-21}$ We found that the number of adjustments in the contralesional forepaw (the movement to adjust a pasta piece with impaired digits) decreased following brain injury (Figure 3c). However, the number of adjustments in the $\mathrm{TBI}+$ rotarod group increased compared with the TBI-only and $\mathrm{TBI}+$ reaching groups (two-way ANOVA, $P<0.05$ ). Differences between the $\mathrm{TBI}+$ reaching and $\mathrm{TBI}$-only groups were not significant (Figure $3 \mathrm{c}$ ).
We next asked whether task-specific rehabilitative training could enhance recovery of that task after TBI and found that mice subjected to single pellet-reaching training exhibited increased scores at 33 days after injury (Figure $3 \mathrm{~d}$; TBI+ reaching versus $\mathrm{TBI}$-only, two-way ANOVA followed by Tukey-Kramer test, $P<0.05)$. In contrast, when mice performed the rotarod task after TBI, the differences between the $\mathrm{TBI}$ + rotarod and $\mathrm{TBI}$-only groups were not significant (Figure 3e).

Bilateral movement but not reaching training promotes CST rewiring after TBI. The intact CST is capable of sprouting fibers that enter the denervated side of the spinal cord and make functional connections that are necessary for motor recovery. ${ }^{4}$ We examined anatomical changes of the CST in the cervical spinal cord (C4-C7) to determine whether rehabilitative training protocols enhanced CST plasticity at day 34 after brain injury. Biotinylated dextran amine (BDA), an anterograde tracer, was injected into the forelimb area of the uninjured sensorimotor cortex to label intact CST fibers, and we counted the number of crossing fibers into each compartment of the denervated side (Figures $4 \mathrm{a}$ and $\mathrm{g}$ ). The number of BDA-labeled crossing fibers increased following TBI (Figures $4 \mathrm{~b}, \mathrm{c}$, and h; TBI-only versus sham, two-way ANOVA followed by Tukey-Kramer test, $P<0.01)$. The TBI + rotarod group showed a significantly higher number of crossing CST fibers compared with the $\mathrm{TBI}$-only or $\mathrm{TBI}+$ reaching group, whereas $\mathrm{TBI}+$ reaching did not enhance CST rewiring compared with the TBI-only group (Figures 4c-e and h). Rotarod alone (without $\mathrm{TBI}$ ) did not increase the number of crossing fibers compared with the sham group (sham + rotarod in Figures $4 b, f$, and h).

Effect of rehabilitative training on cervical cord neuronal activity after TBI. The finding that rehabilitative training enhances crossing CST fibers innervation suggests that neuronal activity in the cervical cord might be important for plasticity and functional recovery. c-Fos, an activity-dependent immediate-early gene, was used as a marker of neuronal activity. ${ }^{22}$ The number of c-Fos-positive cells markedly increased in the $\mathrm{TBI}$ + rotarod group compared with the $\mathrm{TBI}$-only or $\mathrm{TBI}+$ reaching group at day 12 after brain injury (Figures 5a-g; two-way ANOVA followed by Tukey-Kramer test, $P<0.05)$. Reach training slightly increased the number of c-Fos-positive cells, but the result was not statistically different from that in the TBI-only group (Figures $5 a, b, d, e$, and g). Next, we examined anatomical changes of the CST rewiring at the same day 12 to determine whether increased c-Fos-positive cells was a consequence of rewired neuronal network of CST or a prerequisite for the induction of CST rewiring. BDA-labeled crossing CST fibers at day 12 did not increase compared with those at day 33 in each group (Figures 5h-k; two-way ANOVA followed by Tukey-Kramer test, $P<0.05)$. This suggests that the training increases neural activity in the spinal circuit before CST is reorganized.

\section{Discussion}

In this study, we assessed the effectiveness of skilled forelimb repetitive training and bilateral movement training in 

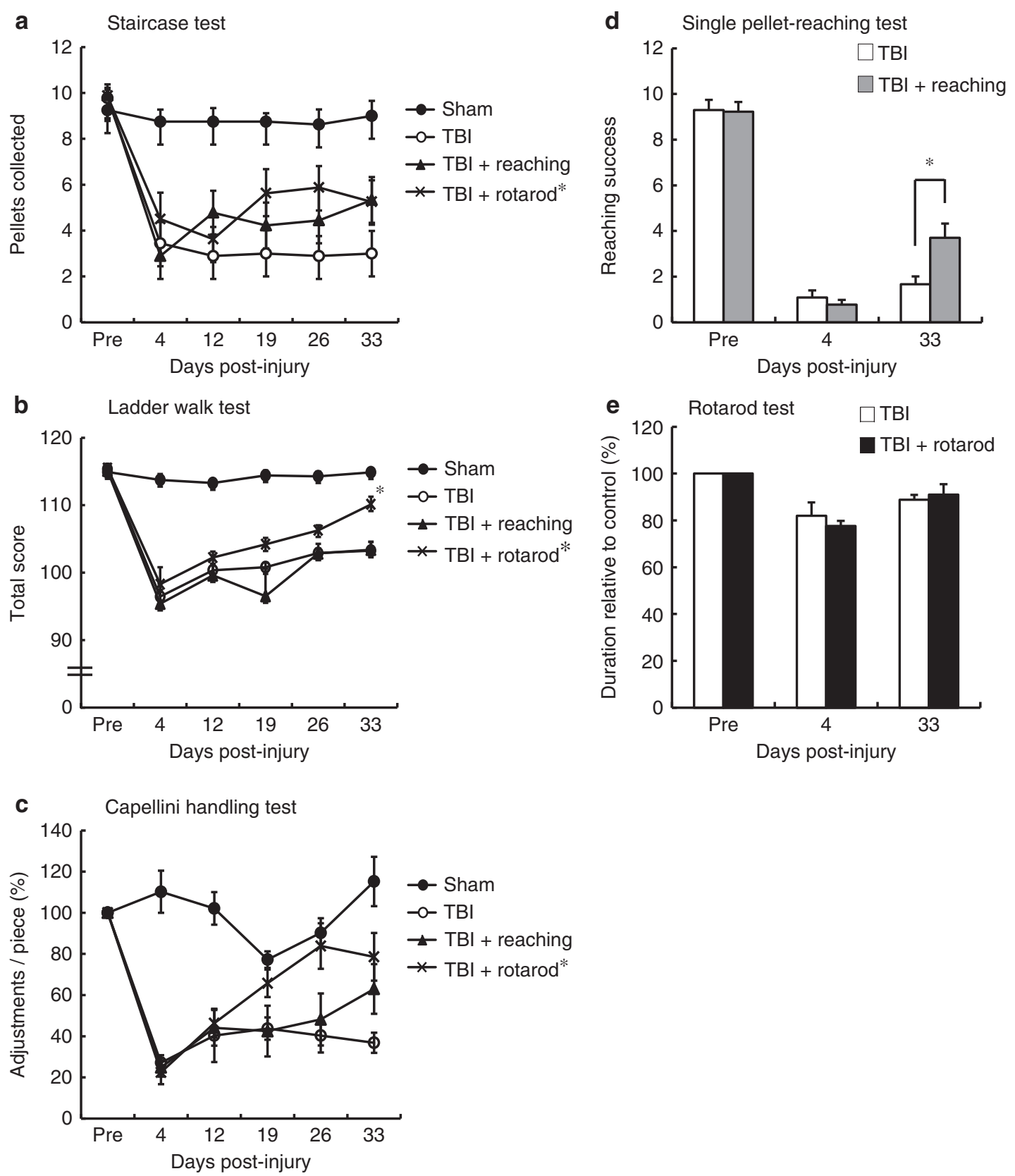

Figure 3 Motor function recovery following rehabilitative training. (a) Staircase test (sham, $n=8 ; \mathrm{TBI}, n=9$; TBI + reaching, $n=9 ; \mathrm{TBI}+$ rotarod, $n=8) ;(\mathbf{b})$ ladder walk test (sham, $n=8$; TBI, $n=11$; TBI + reaching, $n=9$; TBI + rotarod, $n=8$ ); (c) capellini handling test (sham, $n=6$; TBI, $n=9 ; \mathrm{TBI}+$ reaching, $n=9 ; \mathrm{TBI}+$ rotarod, $n=8$ ); (d) single pellet-reaching test (TBI, $n=8$; TBI + reaching, $n=9$ ); and (e) rotarod test (TBI, $n=8$; TBI + rotarod, $n=8)$. Two-way ANOVA or that followed by Tukey-Kramer test, ${ }^{*} P<0.05$

promoting functional recovery in an established TBI model and investigated whether they promoted CST axonal rewiring. The results revealed that bilateral movement training improved motor function recovery and facilitated axonal rewiring, whereas the beneficial effects of simple repetitive training were limited. Many studies have shown enhanced functional recovery by employing one rehabilitative task or a combination

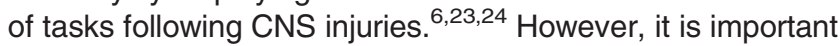
to obtain more detailed information about effective rehabilitative training protocols that enhance motor function and neural rewiring in animal models, as this will be the scientific basis for determining which types of rehabilitative training should be implemented in patients with CNS injuries. It is also important to note that CST plasticity and its underlying mechanisms might differ between mice and humans.

In recent years, a form of bilateral movement training known as active-passive bilateral therapy has been described as an effective neurorehabilitation protocol following CNS-injured patients. ${ }^{11-13}$ Because rotarod training requires rhythm, balance, and coordination of bilateral motor performance, we chose it as a comparable bilateral training protocol to test in rodents. Previous studies have shown that rotarod training 
a

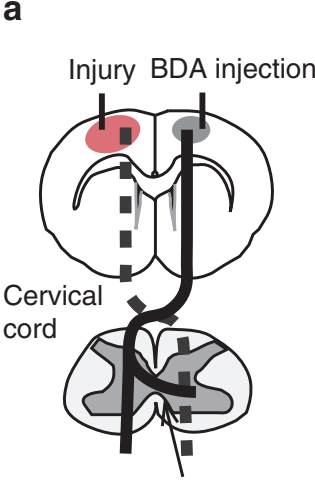

CST crossing fibers
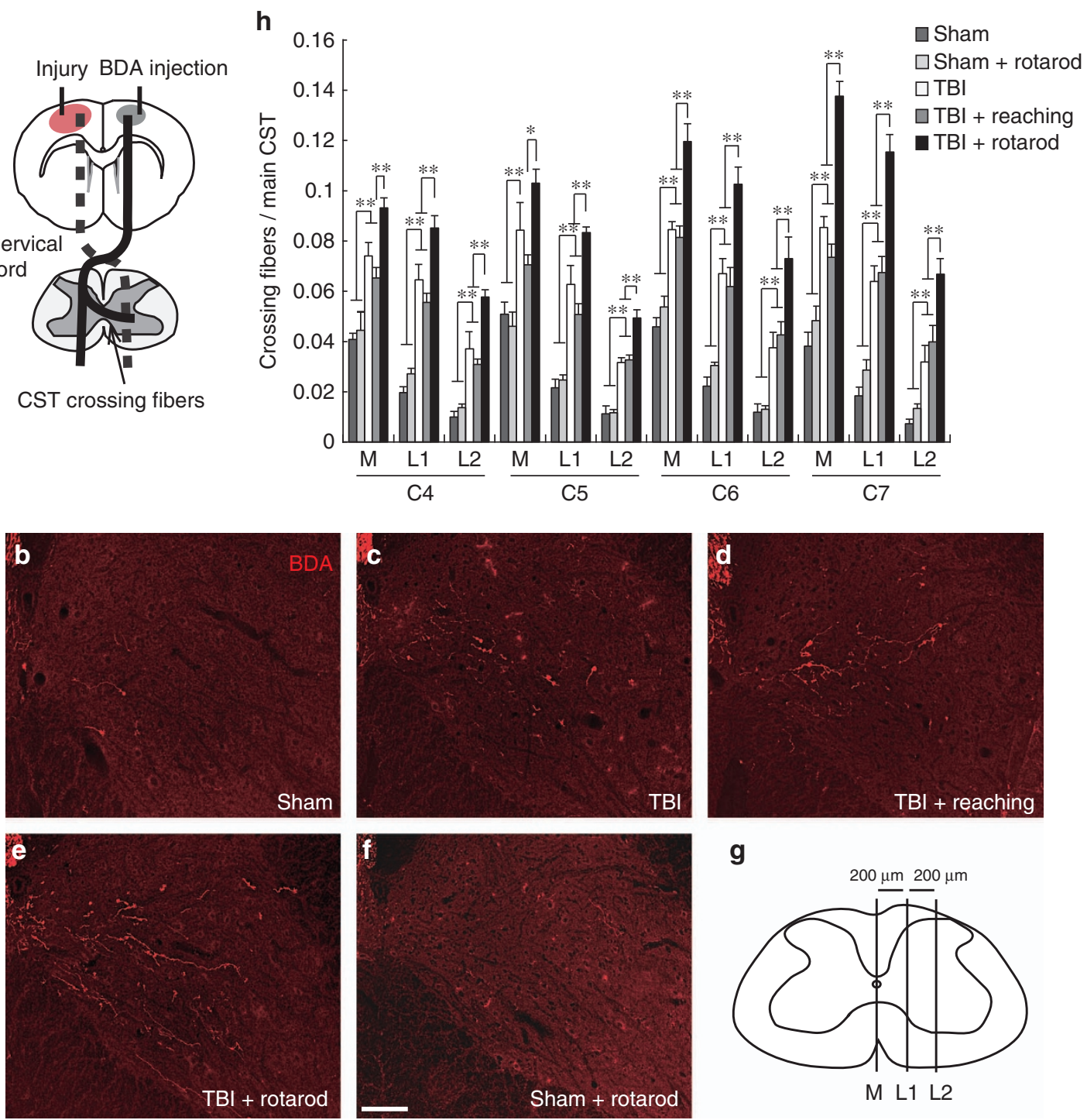

Figure 4 CST rewiring in the cervical cord after rehabilitative training. (a) Diagram of the injury model. The injury to the primary motor cortex leads to the degeneration of corticofugal CST projections (dotted lines). BDA was injected into the contralesional sensorimotor cortex to label intact CST. The arrow shows the rewired crossing fibers from intact CST to the denervated side that are related to functional recovery. $(b-f)$ Representative images of BDA-labeled CST fibers at C7 of the denervated cervical cord in sham (b), TBI (c), TBI + reaching (d), TBI + rotarod (e), and sham + rotarod groups (f) (34 days after TBI). Scale bar: $100 \mu \mathrm{m}$. (g) Spinal cord illustration indicating three vertical lines at 200- $\mu \mathrm{m}$ intervals (M, L1, and L2) to create compartments for counting crossing fibers. (h) The number of crossing CST fibers in each compartment (M, M-L1; L1, L1L2; and L2, lateral to L2) of the denervated side in C4-C7. Two-way ANOVA followed by Tukey-Kramer test, ${ }^{*} P<0.05,{ }^{* *} P<0.01$

enhances the recovery of whole-body balance and hindlimb coordination after stroke, ${ }^{25}$ and our results further indicate that this training is effective in recovering forelimb function following TBI (Figures $3 a-c)$. Although the mechanism of recovery of bilateral therapy in brain-injured patients (or in rodent models) is still unknown, it is thought to restore the balance of inhibitory function within and between hemispheres that is upset by brain injury. ${ }^{11-13}$ Our data further suggest that intraspinal CST rewiring would underlie the recovery. Indeed, we recently demonstrated that intraspinal axonal regeneration of the CST is critical for recovery in a mouse model of TBI. ${ }^{4}$ Consistent with this, promoting rewiring by activating intrinsic regenerative capacity is strongly associated with enhanced functional recovery. ${ }^{17}$ Constraintinduced movement therapy after unilateral CST injury also facilitates CST fiber rewiring and functional recovery. ${ }^{26}$ It should be noted that although reorganization of contralesional CST would be indispensable for the recovery in our severe TBI model in which most of the ipsilesional CST is degenerated, in mild brain injury, ipsilesional spared CST is rewired. ${ }^{27}$ In any cases, although their origin differs between ipsi- and contralesional, the reorganization of CST fibers will be the key neural mechanisms for the recovery by rehabilitating training.

We previously demonstrated that brain-derived neurotrophic factor (BDNF) produced by intraspinal interneurons is a key factor for inducing CST axonal rewiring. ${ }^{4}$ This suggests that bilateral movement training has a beneficial influence on motor performance by modulating a variety of intraspinal systems. ${ }^{28}$ Central pattern generators (CPGs) are 


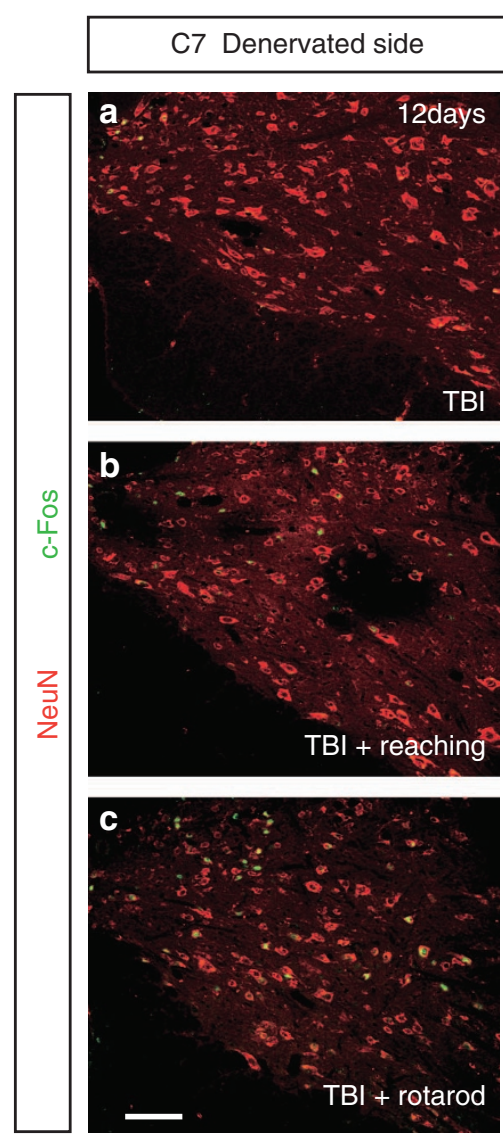

g

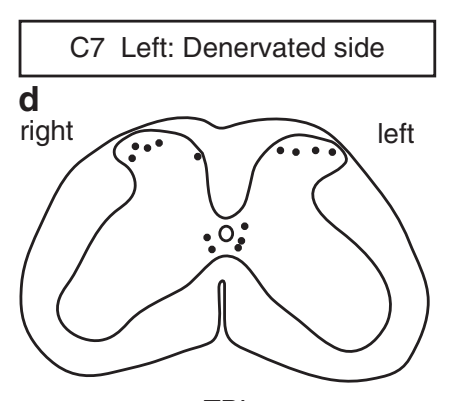

TBI

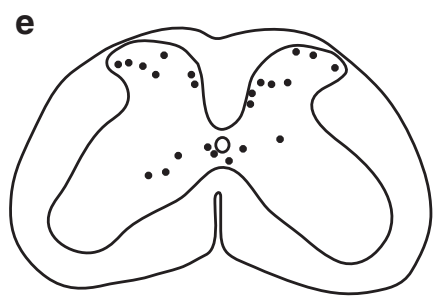

TBI + reaching

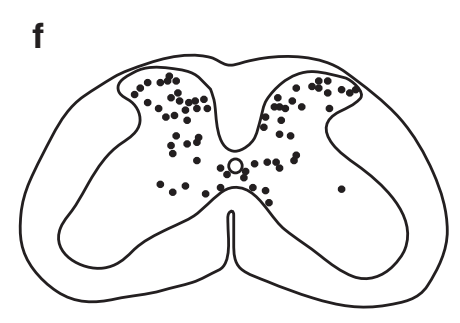

$\mathrm{TBI}+$ rotarod
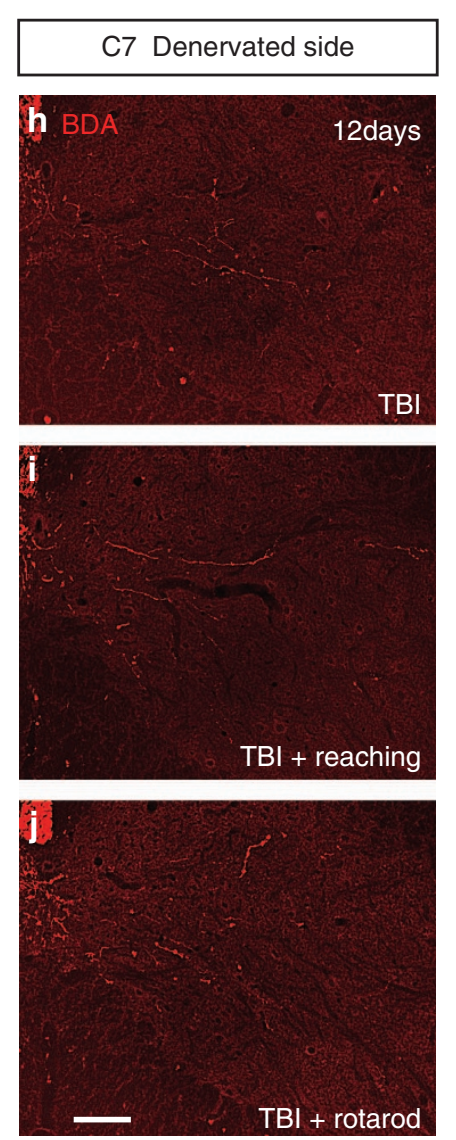

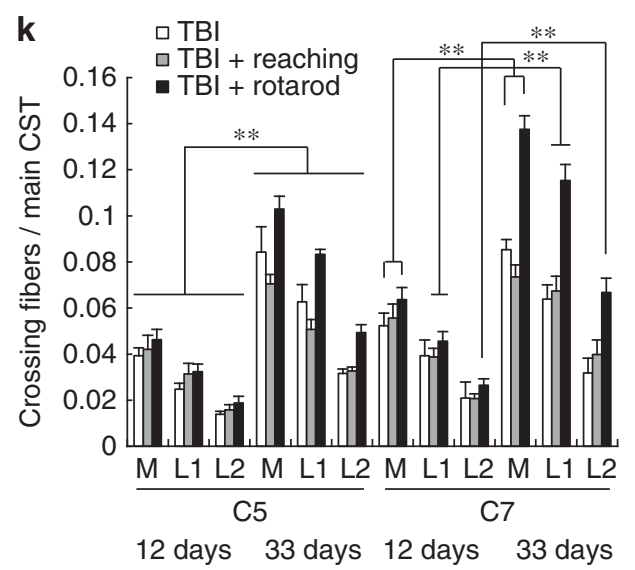

Figure 5 Changes in c-Fos expression and crossing CST fibers in cervical cord after rehabilitative training. (a-c) Representative images of NeuN (red) and c-Fos (green) double staining at $\mathrm{C} 7$ of the denervated side (day 12 after TBI). (a) TBI; (b) TBI + reaching; and (c) TBI + rotarod. Scale bar: $100 \mu$ m. (d and e) Plots showing c-Fos-positive cells localization. (d) TBI; (e) TBI + reaching; and (f) TBI + rotarod. (g) The number of c-Fos-positive cells in the denervated side of C4-C7 (lamina VII, VIII, and X). $(\mathrm{h}-\mathrm{j})$ Representative images of BDA-labeled CST fibers at C7 of the denervted cervical cord in TBI, $n=5$ (h), TBI + reaching, $n=6$ (i), and TBI + rotarod, $n=5$ (j) groups (day 12 after TBI). (k) The number of crossing fibers in each compartment (M, M-L1; L1, L1-L2; and L2, lateral to L2) of the denervated side in C5 and C7 at days 12 and 33 after the injury. Two-way ANOVA followed by Tukey-Kramer test, ${ }^{\star} P<0.05,{ }^{\star \star} P<0.01$

neural networks in the spinal cord responsible for generating rhythmic movements and are important for mediating reciprocal coordination between the right and left sides of the spinal cord. ${ }^{29}$ In particular, commissural interneurons located in laminae VII, VIII, and X of the rodent spinal cord contain the necessary neuronal elements for generating locomotor-like activity. ${ }^{30,31}$ Interestingly, rotarod training increased neuronal activity in laminae VII, VIII, and X of the denervated side (Figures 5c, f, and $\mathrm{g}$ ). Hence, the spinal interneurons in CPGs, including the commissural interneurons, might be involved in rotarod-induced recovery. Rotarod training increased neural activity in the $\mathrm{C} 4-\mathrm{C} 7$ regions (Figures $5 \mathrm{c}, \mathrm{f}$, and $\mathrm{g}$ ), which corresponds to the area where CST rewiring occurred in the denervated side (Figures $4 \mathrm{e}$ and h). Particularly, increase of c-Fos-positive cells and crossing CST fibers in $\mathrm{TBI}$ + rotarod group were higher in the lower 
cervical cord (C7), while increase of them was not obvious in $\mathrm{TBI}$-only and $\mathrm{TBI}+$ reaching groups (Figures $4 \mathrm{e}, \mathrm{h}$ and $5 \mathrm{c}, \mathrm{g}$ ). Lower cervical cord contains a number of motor neurons connecting to distal forelimb muscles, and would be important for distal forelimb movements, for example, grasping. ${ }^{32}$ Thus, rotarod training might strengthen the rewiring and function related to these skilled forelimb movements, especially in the lower spinal cord. Although the molecular mechanism that mediated training-induced rewiring remains unclear, neural activity and its related molecules are possible candidates. Our results showed a correlation of neural activity and CST sprouting as described above. Furthermore, c-Fos expression by rehabilitative training increased in earlier phase than CST sprouting started, suggesting that neural activity might be a prerequisite for the axonal rewiring after brain injury (Figure 5). In regard with neural activity-related molecules, BDNF might be involved in this process. Indeed, BDNF is known to be regulated by neural activity, ${ }^{33,34}$ and increased BDNF expression promotes CST rewiring and new connections onto spared descending interneurons following injury. ${ }^{4,35}$ It should be noted, however, that multiple molecules are hypothesized to be involved in postinjury rewiring. ${ }^{4}$

In contrast to bilateral rotarod training, mice in the single pellet-reaching group did not show improved recovery on any tests except the single pellet test (Figures $3 a-d)$. Although the effect of CST-dependent tasks in rehabilitative training after severe TBI has not been mostly understood, this is in line with studies using other CNS injuries that reported that single pellet-reaching task training enhanced behavioral task performance after the injury ${ }^{36-38}$ but did not facilitate improvement on the staircase test. ${ }^{36}$ Thus, simple repetitive training only enhances task-dependent recovery following brain injury. Consistent with this, reach training following CNS injury has been shown to refine similar movements, but other measures are not improved. ${ }^{39,40}$ Although we did not observe enhanced recovery on the rotarod test in the rotarod training group, this behavioral analysis may not be suitable for evaluating recovery in our TBI model because it has been shown that similar cortical injuries do not cause clear impairment in this test (Figure 3e, day 33). ${ }^{41}$

Not surprisingly, reaching training did not enhance intraspinal CST rewiring (Figures $4 \mathrm{~d}$ and $\mathrm{h}$ ), even though the single pellet-reaching task is considered CST-dependent. ${ }^{42,43}$ García-Alías et al. ${ }^{36}$ demonstrated a similar result; they found that reach training alone does not increase the number of sprouting fibers from the unlesioned lateral and ventral CST following cervical cord injury. In contrast, Starkey et al. ${ }^{44}$ reported that reach training following unilateral pyramidotomy increased crossing CST fibers in C3-C6. The reason for these differences is unknown, but it might be due to variability in the CNS injury models and subsequent spared neural networks. In this study, neuronal activity in the cervical cord, as assessed by c-Fos expression, was not significantly increased by reach training (Figures $5 \mathrm{~b}$, e, and $\mathrm{g}$ ). In the cervical cord, the lower C7-8 spinal cord segments contain motor neurons that activate muscles that control distal forelimb movements required for grasping, whereas neurons located in the upper $\mathrm{C} 4$ segment are associated with proximal forelimb control. $^{32,45}$ In intact rats, single pellet-reaching training alters neuronal structure at C8 but not C4 neurons. ${ }^{46}$
Similarly, we observed that c-Fos-positive cells tended to increase in the denervated side at the lower $\mathrm{C} 7$ but not in the upper C4 (Figure $5 \mathrm{~g}$ ). Taken together, alteration of intraspinal neuronal activity might be insufficient to promote CST rewiring following single pellet-reach training.

This study indicates that the adult CNS has limited but effective endogenous neural plasticity that can be facilitated by rehabilitative training, and the extent of plasticity is dependent on the training protocols. Further studies to elucidate the neural and molecular bases of recovery would support the development of effective rehabilitative training and clinical therapies for brain-injured patients.

\section{Materials and Methods}

Animals. Adult male C57BL/6J mice (8 weeks old, 20-25 g; SLC, Shizuoka, Japan) were group-housed under a 12-h light/dark cycle and received food and water ad libitum. All experimental procedures were approved by the Institutional Animal Care and Use Committee of Osaka University.

Surgery for TBI. Surgical procedures for TBI were performed as described previously. ${ }^{4,14}$ The animals were deeply anesthetized with somnopentyl $(55 \mathrm{mg} / \mathrm{kg}$; Kyoritsu Seiyaku, Tokyo, Japan) and stabilized in a stereotaxic frame (Muromachi, Tokyo, Japan). The skull and dura over the sensorimotor cortex contralateral to the preferred paw (see the methods below) were removed using a drill with a $4.0-\mathrm{mm}$ diameter (at the center, $0.5 \mathrm{~mm}$ anterior, $2.0 \mathrm{~mm}$ lateral to bregma), and cortical injury was induced using a pneumatic impact device (diameter $3.0 \mathrm{~mm}$, depth $1.0 \mathrm{~mm}$; Amscien Instruments, Richmond, VA, USA). Thereafter, the skull was replaced, and the scalp was sutured.

Experimental design. The experimental design is summarized in Figure 2. Animals were trained on behavioral tests for the following periods before injury (single pellet test and staircase test, 2 weeks; ladder walk test, 1 week; rotarod test, 3 days; capellini handling test, training not required). The baseline values of all behavioral tests were evaluated 1 day before the injury. Thereafter, the staircase, ladder walk, and capellini handling tests were performed 4, 12, 19, 26, and 33 days after brain injury. The single pellet and rotarod tests were performed on days 4 and 33. Regarding rehabilitative training, reach training or rotarod training was conducted for 4 or 3 days before brain injury, respectively. Thereafter, rehabilitative training was performed for 4 weeks, starting 5 days after injury (Figure 2).

\section{Rehabilitative procedures and behavioral tests.}

Single pellet-reaching training and test: The single pellet-reaching task is a simple repetitive training protocol used to test motor function. ${ }^{47}$ The single pelletreaching box was made of clear Plexiglas $(13 \mathrm{~cm}$ long $\times 7 \mathrm{~cm}$ wide $\times 13 \mathrm{~cm}$ high) with vertical slits in the center and side of the front wall $(7$ and $8 \mathrm{~mm}$ wide, respectively) and a 3-cm-wide shelf mounted $1 \mathrm{~cm}$ above the ground in front of the slit. A small metal bar (height $2 \mathrm{~mm}$ ) was adhered to the base of the reaching window to create a barrier. This bar prevented animals from simply pushing the pellet into their mouth and forced them to grasp the pellet in their paw and lift it over the bar. The pellet was placed on the shelf $1 \mathrm{~cm}$ from the slit. The vertical slit in the center was used for determining the preferred paw. ${ }^{48}$ During the initial phase, mice used both paws to reach pellets in front of the central slit. Paw preference was determined when the animal showed $>70 \%$ preference over 20 reach attempts.

The animals were trained for 2 weeks before TBI to take pellets $(20 \mathrm{mg}$, chocolate flavor; Bioserve, Frenchtown, NJ, USA) in the Plexiglas box (TBI, $n=8$; $\mathrm{TBI}+$ reaching, $n=9$ ). Food intake was restricted the night before the test. Animals were tested in the box for 20 pellets in each trial. A reach was considered successful when a mouse moved the pellet to its mouth and ate it. An attempt was counted as a miss if the animal knocked away or dropped the pellet after grasping it. The performance of each animal was scored as the number of successful retrievals. The baseline value was scored as the mean of three trials 1 day before the injury.

At 5 days after TBI, mice were subjected to single pellet training rehabilitation consisting of 60 single pellets atempts or 30 min, whichever came first, 6 times per week for 4 weeks. Food intake was restricted to $80-85 \%$ per day, and body weight was maintained at approximately $85-95 \%$ of baseline value throughout the experiment. 
Rotarod training and test: The rotarod is used to assess motor coordination in rodents ${ }^{24}$ and served as an effective bilateral motor training protocol in this study. Animals were placed on a rotating rod (diameter $30 \mathrm{~mm}$ ) that accelerated from 4 to 40 r.p.m. within a 5 -min period. ${ }^{49}$ The animals were trained two times a day for 3 days before injury (TBI, $n=8 ; \mathrm{TBI}+$ rotarod, $n=8$ ). Total time was counted until the mouse fell off the rod or gripped and spun around two times. The baseline value was scored as the mean of three trials 1 day before TBI. The data were expressed as the percentage of duration relative to baseline.

For rotarod rehabilitation, animals trained for $30 \mathrm{~min}, 6$ days per week, starting 5 days after TBI. Because motor function was impaired, animals were initially run at a constant 20 r.p.m. speed on the first day, and speed was gradually increased to 25 r.p.m. at 2-4 days, 30 r.p.m. at 5-6 days, and 35 r.p.m. from 4 weeks on.

Staircase test: The staircase test was used to assess skilled forelimb reaching. ${ }^{50}$ At 2 weeks before the injury, mice were trained to reach pellets on the staircase (Melquest, Toyama, Japan) for $30 \mathrm{~min}$ (sham, $n=8$; TBI, $n=9$; TBI + reaching, $n=9$; $\mathrm{TBI}+$ rotarod, $n=8$ ). Each staircase consisted of seven steps with two food pellets placed on each step, and the number of pellets retrieved in $30 \mathrm{~min}$ was counted for each side. The pellets on the first and second stairs were not counted because animals could retrieve them using only their tongue. Food intake was restricted the night before the test, and the baseline value was assessed 1 day before TBI.

Ladder walk test: The ladder walk test was used to assess skilled walking and measure forelimb placing, stepping, and interlimb coordination. ${ }^{17}$ The animals were placed on a 1-m runway comprised of randomly spaced metal rungs (6$18 \mathrm{~mm}$ intervals) $15 \mathrm{~cm}$ above the ground (sham, $n=8$; TBI, $n=11$; TBI + reaching, $n=9$; TBI + rotarod, $n=8$ ). The mice were video recorded in slow motion as they walked across the 1-m runway (Sony Handycam: Sony, Tokyo, Japan), and foot faults for each forepaw were counted during continuous steps. ${ }^{17}$ The animals were trained for 1 week (each for $10 \mathrm{~min}$ ) before injury and were tested three times per session after training. The baseline value was assessed 1 day before the injury.

Capellini handling test: The capellini handling test was used to characterize forepaw dexterity while eating a piece of pasta. ${ }^{18,19}$ The animals were placed on a mirror in a clear box (sham, $n=6 ; \mathrm{TBI}, n=9 ; \mathrm{TBI}+$ reaching, $n=9 ; \mathrm{TBI}+$ rotarod, $n=8$ ). Uncooked pasta pieces (diameter $0.9 \mathrm{~mm}$ and length $26 \mathrm{~mm}$ ) were eaten in three sequential trials after training. The number of forepaw adjustments made while eating the pasta was counted using slow-motion video $(\sim 10 \times$ zoom). An adjustment was defined as any distinct removal and replacement of the paw or digits on the pasta (re-contacts the previous piece, regrips the piece, or extension-flexion or abduction-adduction of the digits). Cases when the paw slid across the pasta piece with no digit movement or repositioning were not counted, nor were paw movements that did not contact the pasta. Because the test was performed the same day as the staircase test, food intake was restricted. The baseline value was scored as the mean of three trials 1 day before injury. The data were expressed as the percentages of duration relative to baseline.

Immunohistochemistry. The animals were deeply anesthetized and transcardially perfused with $4 \%$ paraformaldehyde (PFA) in $0.1 \mathrm{M}$ phosphate buffer $(\mathrm{PB})$. Following perfusion, the brain and spinal cord were postfixed in the same fixative solution at $4{ }^{\circ} \mathrm{C}$ overnight and subsequently transferred to $30 \%$ sucrose in phosphate-buffered saline (PBS) at $4{ }^{\circ} \mathrm{C}$ overnight. The brain and spinal cord were cut into $20-\mu \mathrm{m}$-thick coronal sections using a cryostat and placed on MAS-coated glass slides (Matsunami, Osaka, Japan). For immunohistochemistry, the sections were immersed for $5 \mathrm{~min} \times 3$ in PBS and blocked with $5 \%$ bovine serum albumin/0.1-0.3\% Triton X-100/PBS for $1 \mathrm{~h}$. Subsequently, the sections were incubated with primary antibodies overnight at room temperature (RT) or at $4^{\circ} \mathrm{C}$. Mouse anti-neuronal nuclei (NeuN) (1:100; Millipore, Temecula, CA, USA), rabbit anti-c-Fos (1: 1000; Santa Cruz Biotechnology, Santa Cruz, CA, USA), and rabbit anti-GFP (1:1000; Invitrogen, Carlsbad, CA, USA) were used as primary antibodies. The sections were then washed three times with PBSTween-20, followed by incubation with secondary antibodies for $1 \mathrm{~h}$ at $\mathrm{RT}$ in the dark. Goat-anti-rabbit or mouse IgG Alexa 488 or 568 (1:500; Invitrogen) were used as secondary antibodies. The sections were counterstained with Neurotracer Nissl (1:1000; Invitrogen). For BDA labeling, the sections were immersed three times in PBS and incubated in $0.3 \%$ Triton X-100/PBS for $1 \mathrm{~h}$, followed by incubation with Alexa Fluor 568-conjugated streptavidin (1:400; Invitrogen) for $2 \mathrm{~h}$ at RT. All images were acquired with a fluorescence microscope (Keyence, Biorevo BZ-9000, Osaka, Japan) or a confocal laser-scanning microscope (Olympus FluoView FV1000, Tokyo, Japan).

Lesion volume measurement. At 34 days after $\mathrm{TBI}$, the animals were deeply anesthetized and perfused with $4 \%$ PFA (TBI, $n=5$; TBI + reaching, $n=5 ; \mathrm{TBI}+$ rotarod, $n=5$ ). The brains were sectioned at 40 per $200 \mu \mathrm{m}$ in the coronal plane at the level of the forelimb area (bregma +1.2 to $-1.0 \mathrm{~mm}$ ) and stained with Nissl. The lesion volume of each animal was calculated as a percentage by dividing the volume of the injured hemisphere by that of the intact contralesional hemisphere using the ImageJ software (National Institutes of Health, Bethesda, MD, USA). ${ }^{51}$

Anterograde tracing of CST fibers. The descending CST fibers were labeled with the anterograde tracer BDA (MW, 10000; 10\% BDA in PBS; Invitrogen) 2 weeks before the animals were killed. BDA injection procedures were performed as described previously. ${ }^{4}$ The animals were anesthetized and stabilized in a stereotaxic frame. The corresponding skull area on the contralesional side was opened, and 2.7 $\mu \mathrm{l}$ BDA was stereotactically infused into the forelimb area of the sensorimotor cortex at three sites (coordinates from bregma: $0 \mathrm{~mm}$ anterior/ $1.0 \mathrm{~mm}$ lateral, $0.5 \mathrm{~mm}$ anterior $/ 1.0 \mathrm{~mm}$ lateral, and $0.5 \mathrm{~mm}$ anterior $/ 1.5 \mathrm{~mm}$ lateral, all at a depth of $0.5 \mathrm{~mm}$ ) using a glass capillary attached to a microsyringe.

CST fibers' quantification. The numbers of midline-crossing BDA-labeled CST fibers were counted at $\times 400$ magnification in each section (20 sections per segment, C4-C7; sham, $n=5$; TBI, $n=5$; TBI + reaching, $n=5$; TBI + rotarod, $n=5$; sham + rotarod, $n=4$; for Figure $4 \mathrm{~h}$ : TBI, $n=5$; TBI + reaching, $n=6$; TBI + rotarod, $n=5$; for Figure $5 \mathrm{k}$ ). The numbers of CST fibers in the denervated side of the cervical cord were counted in each of three compartments, which were defined by three vertical lines: $M, L 1$, and L2. The first vertical line M was drawn through the central canal, and $L 1$ and $L 2$ were parallel to $M$ at distances of 200 and $400 \mu \mathrm{m}$, respectively (Figure 4f). To correct for tracing differences among individual animals, the results were normalized to the mean number of BDA-labeled CST fibers in the dorsal funiculus at $\mathrm{C} 1$ (three serial transverse sections ${ }^{15}$ ).

Quantification of c-Fos-positive neurons. Animals at 12 days after injury were used to quantify $\mathrm{c}$-Fos-expressing neurons in the cervical cord after training by same protocols (TBI, $n=4 ; \mathrm{TBI}+$ reaching, $n=4 ; \mathrm{TBI}+$ rotarod, $n=4)$. To ensure adequate time for $c$-Fos expression, animals were returned to their cage after training and allowed to rest for $1 \mathrm{~h}$. Then, the mice were perfused, and the tissues were processed as described above. c-Fos-positive neurons were counted in 10 sections per cervical level (C4-C7), and the mean number was calculated in each animal. Neurons were identified by double staining with NeuN. They were quantified within lamina VII, VIII, and X of the denervated side in the cervical cord. The localization of c-Fos-positive cells was plotted with Neurolucida (MBF Bioscience, Williston, VT, USA).

Retrograde transsynaptic tracing with PRV. The PRV was used as a transsynaptic retrograde tracer to verify neuronal connections between the motor cortex and the impaired forelimb. ${ }^{15}$ The day after the final behavioral test, animals were anesthetized and injected with PRV expressing EGFP under a cytomegalovirus promoter (PRV-152, gift from Dr. JP Card and LW Enquist) at five sites $\left(2 \mu \mathrm{l}\right.$ per site, titer: $\left.4.2 \times 10^{8} \mathrm{PFU} / \mu \mathrm{l}\right)$ in the extensor muscle of the impaired forelimb by using a glass capillary attached to a microsyringe. After $120 \mathrm{~h}$, the animals were perfused, and the tissues were processed as described above. The brains were sectioned at $20 \mu \mathrm{m}$ in the coronal plane at the level of the forelimb area of the motor cortex (bregma +1.2 to $-1.0 \mathrm{~mm}$ ), and the sections were stained with an anti-GFP antibody and Neurotracer Nissl.

Statistics. Lesion volume was statistically analyzed by one-way ANOVA. Behavioral tests were analyzed by two-way ANOVA, and Tukey-Kramer post hoc test was applied when appropriate. CST fibers and c-Fos-positive cells results were analyzed using two-way ANOVA followed by Tukey-Kramer test. All data are represented as mean \pm S.E.M., and statistical significance was accepted at $P<0.05$.

\section{Conflict of Interest}

The authors declare no conflict of interest. 
Acknowledgements. We thank Dr. J Patrick Card (University of Pittsburgh) and Lynn W Enquist (Princeton University) for their kind gift of PRV. This work was supported by Core Research for Evolutional Science and Technology (CREST) from Japan Science and Technology Agency (JST).

1. Jacobsson LJ, Westerberg M, Söderberg S, Lexell J. Functioning and disability 6-15 years after traumatic brain injuries in northern Sweden. Acta Neurol Scand 2009; 120: 389-395.

2. Nakayama H, Jorgensen HS, Raaschou HO, Olsen TS. Compensation in recovery of upper extremity function after stroke: the Copenhagen stroke study. Arch Phys Med Rehabil 1994; 75: 852-857.

3. Lemon RN. Cortical control of the primate hand. Exp Physiol 1993; 78: 263-301.

4. Ueno M, Hayano $Y$, Nakagawa $H$, Yamashita $T$. Intraspinal rewiring of the corticospinal tract requires target-derived brain-derived neurotrophic factor and compensates lost function after brain injury. Brain 2012; 135: 1253-1267.

5. Dobkin BH. Motor rehabilitation after stroke, traumatic brain, and spinal cord injury: common denominators within recent clinical trials. Curr Opin Neurol 2009; 22: 563-569.

6. Conner JM, Chiba AA, Tuszynski MH. The basal forebrain cholinergic system is essential for cortical plasticity and functional recovery following brain injury. Neuron 2005; 46: 173-179.

7. Ramanathan D, Conner JM, Tuszynski MH. A form of motor cortical plasticity that correlates with recovery of function after brain injury. Proc Natl Acad Sci USA 2006; 103 : $11370-11375$.

8. French B, Thomas LH, Leathley MJ, Sutton CJ, McAdam J, Foorster A et al. Repetitive task training for improving functional ability after stroke. Cochrane Database Syst Rev 2007; 4: CD006073.

9. Woldag $\mathrm{H}$, Waldmann $\mathrm{G}$, Heuschkel $\mathrm{G}$, Hummelsheim $\mathrm{H}$. Is the repetitive training of complex hand and arm movements beneficial for motor recovery in stroke patients? Clin Rehabil 2003; 17: 723-730.

10. Whitall J, McCombe Waller S, Silver KH, Macko RF. Repetitive bilateral arm training with rhythmic auditory cueing improves motor function in chronic hemiparetic stroke. Stroke 2000; 31: 2390-2395.

11. Cauraugh JH, Summers JJ. Neural plasticity and bilateral movements: a rehabilitation approach for chronic stroke. Prog Neurobiol 2005; 75: 309-320.

12. Luft AR, McCombe-Waller S, Whitall J, Forrester LW, Macko R, Sorkin JD et al. Repetitive bilateral arm training and motor cortex activation in chronic stroke: a randomized controlled trial. JAMA 2004; 292: 1853-1861.

13. Stinear CM, Barber PA, Coxon JP, Fleming MK, Byblow WD. Priming the motor system enhances the effects of upper limb therapy in chronic stroke. Brain 2008; 131: 1381-1390.

14. Omoto S, Ueno M, Mochio S, Takai T, Yamashita T. Genetic deletion of paired immunoglobulin-like receptor $B$ does not promote axonal plasticity or functional recovery after traumatic brain injury. J Neurosci 2010; 30: 13045-13052.

15. Liu Z, Zhang X, Savant-Bhonsale S, Chopp M. Contralesional axonal remodeling of the corticospinal system in adult rats after stroke and bone marrow stromal cell treatment. Stroke 2008; 39: 2571-2577.

16. Baird AL, Meldrum A, Dunnett SB. The staircase test of skilled reaching in mice. Brain Res Bull 2001; 54: 243-250.

17. Smith JM, Lunga $P$, Story D, Harris N, Le Belle J, James MF et al. Inosine promotes recovery of skilled motor function in a model of focal brain injury. Brain 2007; 130: 915-925.

18. Metz GA, Whishaw IQ. Cortical and subcortical lesions impair skilled walking in the ladder rung walking test: a new task to evaluate fore- and hindlimb stepping, placing and co-ordination. J Neurosci Methods 2002; 115: 169-179.

19. Allred RP, Adkins DL, Woodlee MT, Husbands LC, Maldonado MA, Kane JR et al. The vermicelli handling test: a simple quantitative measure of dexterous forepaw function in rat. J Neurosci Methods 2008; 170: 229-244.

20. Tennant KA, Asay AL, Allred RP, Ozbum AR, Kleim JA, Jones TA. The vermicelli and capellini handling tests: simple quantitative measures of dexterous forepaw function in rats and mice. J Vis Exp 2010; 41: e2076.

21. Whishaw IQ, Coles BL. Varieties of paw and digit movement during spontaneous food handling in rats: postures, bimanual coordination, preferences, and the effect of forelimb cortex lesions. Behav Brain Res 1996; 77: 135-148.

22. Flavell SW, Greenberg ME. Signaling mechanisms linking neuronal activity to gene expression and plasticity of the nervous system. Annu Rev Neurosci 2008; 31: 563-590.

23. Jones TA, Chu CJ, Grande LA, Gregory AD. Motor skills training enhances lesion-induced structural plasticity in the motor cortex of adult rats. J Neurosci 1999: 19: 10153-10163.

24. Will B, Galani R, Kelche C, Rosenzweig MR. Recovery from brain injury in animals: relative efficacy of environmental enrichment, physical exercise or formal training (1990-2002). Prog Neurobiol 2004; 72: 167-182.

25. Ding Y, Li J, Lai Q, Rafols JA, Luan X, Clark J et al. Motor balance and coordination training enhances functional outcome in rat with transient middle cerebral artery occlusion. Neuroscience 2004; 123: 667-674.

26. Maier IC, Baumann K, Thallmair M, Weinmann O, Scholl J, Schwab ME. Constraintinduced movement therapy in the adult rat after unilateral corticospinal tract injury. J Neurosci 2008; 28: 9386-9403.
27. Starkey ML, Bleul C, Zörner B, Lindau NT, Mueggler T, Rudin M et al. Back seat driving: hindlimb corticospinal neurons assume forelimb control following ischaemic stroke. Brain 2012; 135: 3265-3281.

28. Wolpaw JR. The complex structure of a simple memory. Trends Neurosci 1997; 20: 588-594.

29. Quinland KA, Kiehn O. Segmental, synaptic actions of commissural interneurons in the mouse spinal cord. J Neurosci 2007; 27: 6521-6530.

30. Kiehn 0 , Kjaerulff $O$. Distribution of central pattern generators for rhythmic motor outputs in the spinal cord of limbed vertebrates. Ann N Y Acad Sci 1998; 860: 110-129.

31. Kjaerulff $\mathrm{O}$, Kiehn $\mathrm{O}$. Distribution of networks generating and coordinating locomotor activity in the neonatal rat spinal cord: a lesion study. J Neurosci 1996; 16: 5777-5794.

32. McKenna JE, Prusky GT, Whishaw IQ. Cervical motoneuron topography reflects the proximodistal organization of muscle and movements of the rat forelimb: a retrograde carbocyanine dye analysis. J Comp Neurol 2000; 419: 286-296.

33. Martinowich K, Manji H, Lu B. New insights into BDNF function in depression and anxiety. Nat Neurosci 2007; 10: 1089-1093.

34. Zafra F, Hengerer B, Leibrock J, Thoenen $\mathrm{H}$, Lindholm D. Activity dependent regulation of BDNF and NGF mRNAs in the rat hippocampus is mediated by non-NMDA glutamate receptors. EMBO J 1990; 9: 3545-3550.

35. Vavrek R, Girgis J, Tetzlaff W, Hiebert GW, Fouad K. BDNF promotes connections of corticospinal neurons onto spared descending interneurons in spinal cord injured rats. Brain 2006; 129: 1534-1545.

36. García-Alías G, Barkhuysen S, Buckle M, Fawcett JW. Chondroitinase ABC treatment opens a window of opportunity for task-specific rehabilitation. Nat Neurosci 2009; 12: $1145-1151$.

37. Girgis J, Merrett D, Kirkland S, Metz GAS, Fouad K. Reaching training in rats with spinal cord injury promotes plasticity and task specific recovery. Brain 2007; 130: 2993-3003.

38. Ploughman M, Attwood Z, White N, Doré JJ, Corbett D. Endurance exercise facilitates relearning of forelimb motor skill after focal ischemia. Eur J Neurosci 2007; 25: 3453-3460.

39. Krajacic A, Ghosh M, Puentes R, Pearse DD, Fouad K. Advantages of delaying the onset of rehabilitative reaching training in rats with incomplete spinal cord injury. Eur $J$ Neurosci 2009; 29: 641-651.

40. Krajacic A, Weishupt N, Girgis J, Tetzlaff W, Fouad K. Training-induced plasticity in rats with cervical spinal cord injury: effects and side effects. Behav Brain Res 2010; 214: 323-331.

41. Tabuse M, Yaguchi M, Ohata S, Kawase T, Toda M. A simple behavioral test for locomotor function after brain injury in mice. J Clin Neurosci 2010; 17: 1412-1416.

42. Whishaw IQ, Gorny B, Sarna J. Paw and limb use in skilled and spontaneous reaching after pyramidal tract, red nucleus and combined lesions in the rat: behavioral and anatomical dissociations. Behav Brain Res 1998; 93: 167-183.

43. Whishaw IQ, Pellis SM, Gorny B, Kolb B, Tetzlaff W. Proximal and distal impairments in rat forelimb use in reaching follow unilateral pyramidal tract lesions. Behav Brain Res 1993; 56: $59-76$.

44. Starkey ML, Bleul C, Maier IC, Schwab ME. Rehabilitative training following unilateral pyramidotomy in adults rats improves forelimb function in a non-task specific way. Exp Neurol 2011; 232: 81-89.

45. Callister RJ, Brichta AM, Peterson EH. Quantitative analysis of cervical musculature in rats: histochemical composition and motor pool organization. II. Deep dorsal muscles. J Comp Neurol 1987; 255: 369-385.

46. Wang L, Conner JM, Rickert L, Tuszynski MH. Structural plasticity within highly specific neuronal populations identifies a unique parcellation of motor learning in the adult brain. Proc Natl Acad Sci USA 2011; 108: 2545-2550.

47. Miklaeva EI, Whishaw IQ. HemiParkinson analogue rats display active support in good limbs versus passive support in bad limbs on a skilled reaching task of variable height. Behav Neurosci 1996; 110: 117-125.

48. Xu T, Yu X, Perlik AJ, Tobin WF, Zweig JA, Tennant $\mathrm{K}$ et al. Rapid formation and selective stabilization of synapses for enduring motor memories. Nature 2009; 462: 915-919.

49. Lee HJ, Kim KS, Kim EJ, Choi HB, Lee KH, Park IH et al. Brain transplantation of immortalized human neural stem cells promotes functional recovery in mouse intracerebral hemorrhage stroke model. Stem Cells 2007; 25: 1204-1212

50. Montoya CP, Campbell-Hope LJ, Pemberton KD, Dunnett SB. The 'staircase test': a measure of independent forelimb reaching and grasping abilities in rats. $J$ Neurosci Methods 1991; 36: 219-228.

51. Swanson RA, Morton TM, Tsao-Wu G, Savalos RA, Davidson C, Sharp FR. A semiautomated method for measuring brain infarct volume. J Cereb Blood Flow Metab 1990; 10: 290-293

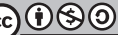

Cell Death and Disease is an open-access journal published by Nature Publishing Group. This work is licensed under a Creative Commons Attribution-NonCommercialShareAlike 3.0 Unported License. To view a copy of this license, visit http://creativecommons.org/licenses/by-nc-sa/3.0/ 